\title{
Correction to: Beta diversity as an indicator of priority areas for Myrtaceae assemblage conservation in Subtropical Araucaria Forest
}

\author{
Juliano Pereira Gomes ${ }^{1}$ (D) - Lilian lara Bet Stedille ${ }^{1}$ (D) . \\ Jaçanan Eloisa de Freitas Milani ${ }^{2}$ (D) Karina Montibeller-Silva ${ }^{1}$ (D) . \\ Adelar Mantovani ${ }^{1}$ (D) . Roseli Lopes da Costa Bortoluzzi ${ }^{1}$ (D)
}

Published online: 10 February 2020

(c) Springer Nature B.V. 2020

\section{Correction to: Biodiversity and Conservation https://doi.org/10.1007/s10531-020-01940-8}

In the original publication of the article, Table 1 was published with few errors and the Acknowledgement statement was inadvertently omitted.

The corrected table and acknowledgement statement is provided in this Correction.

Acknowledgements The authors would like to thank the Santa Catarina State University and the Maintenance Support Fund, as well as the Development of Higher Education in Santa Catarina for graduate scholarships to J.P.G. and K.M.S., the Coordenação de Aperfeiçoamento de Pessoal de Nível Superior (CAPES) for graduate scholarships to L.I.B.S., and the Fundação de Amparo à Pesquisa e Inovação of Santa Catarina (FAPESC).

The original article can be found online at https://doi.org/10.1007/s10531-020-01940-8.

Juliano Pereira Gomes

julianopgomes7@gmail.com

1 Uso e Conservação dos Recursos Florestais (UCRF), Universidade do Estado de Santa Catarina, Avenida Luíz de Camões, 2090, Conta Dinheiro, Lages 88520-000, Brasil

2 Instituto de Ciências Agrárias e Ambientais, Universidade Federal de Mato Grosso, Rua Fernando Corrêa da Costa, 2367, Boa Esperança, Cuiabá, MT 78068-600, Brasil 
Table 1 Features of the sampled areas in the Caveiras and Pelotas River Basins, Subtropical Araucaria Forest, Southern Plateau of Santa Catarina, Brazil. Bioclimatic data. Source: Worldclim2 (2018)

\begin{tabular}{|c|c|c|c|c|c|}
\hline \multirow[t]{2}{*}{ Locations } & \multirow[t]{2}{*}{ Coordinates } & \multicolumn{3}{|l|}{ Mean } & \multirow[t]{2}{*}{ SAF Formations } \\
\hline & & $\mathrm{P}(\mathrm{mm})$ & $\mathrm{T}\left({ }^{\circ} \mathrm{C}\right)$ & $\mathrm{A}(\mathrm{m})$ & \\
\hline SJC—São José do Cerrito ${ }^{a}$ (Private Property) & $\begin{array}{l}27^{\circ} 43^{\prime} \mathrm{S} \\
50^{\circ} 36^{\prime} \mathrm{W}\end{array}$ & 1560 & 16.5 & 798 & Montane \\
\hline LGS_Lages ${ }^{\mathrm{a}}$ (Private Property) & $\begin{array}{l}27^{\circ} 51^{\prime} \mathrm{S} \\
50^{\circ} 09^{\prime} \mathrm{W}\end{array}$ & 1505 & 15.8 & 1018 & Upper-Montane \\
\hline URA-Urupema ${ }^{\mathrm{a}}$ (Conservation Unit) & $\begin{array}{l}27^{\circ} 55^{\prime} \mathrm{S} \\
49^{\circ} 52^{\prime} \mathrm{W}\end{array}$ & 1683 & 13.4 & 1502 & Upper-Montane \\
\hline SJM—São Joaquim ${ }^{\mathrm{b}}$ (Private Property) & $\begin{array}{ll}28^{\circ} 28^{\prime} \mathrm{S} \\
50^{\circ} 04^{\prime} \mathrm{W}\end{array}$ & 1634 & 15.4 & 903 & Montane \\
\hline BJS_-Bom Jardim da Serra ${ }^{b}$ (Private Property) & $\begin{array}{l}28^{\circ} 19^{\prime} \mathrm{S} \\
49^{\circ} 39^{\prime} \mathrm{W}\end{array}$ & 1685 & 14.1 & 1209 & Upper-Montane \\
\hline URI-Urubici ${ }^{\mathrm{b}}$ (Conservation Unit) & $\begin{array}{l}28^{\circ} 07^{\prime} \mathrm{S} \\
49^{\circ} 28^{\prime} \mathrm{W}\end{array}$ & 1752 & 12.9 & 1647 & Upper-Montane \\
\hline
\end{tabular}

River Basins (Caveiras River ${ }^{\mathrm{a}}$-Pelotas River ${ }^{\mathrm{b}}$ ); Mean annual precipitation (P), Mean annual temperature (T), Altitude a.s.l. (A); Forest Formations (IBGE 2012)

Publisher's Note Springer Nature remains neutral with regard to jurisdictional claims in published maps and institutional affiliations. 